\title{
AN INTERACTIVE ANALYSIS OF 3D TECHNOLOGY AND ITS VAST APPLICATION TO HUMAN
}

\author{
Orelaja Oluseyi A. ${ }^{\text {a,c,*, }}$ \\ ${ }^{(a)}$ Department Mechanical Engineering, \\ Moshood Abiola Polytechnic, \\ Abeokuta Ogun State, Nigeria. \\ ${ }^{(c)}$ School of Mechanical Engineering, \\ Southeast University, \\ Nanjing, Jiangsu 211189, \\ P. R. China
}

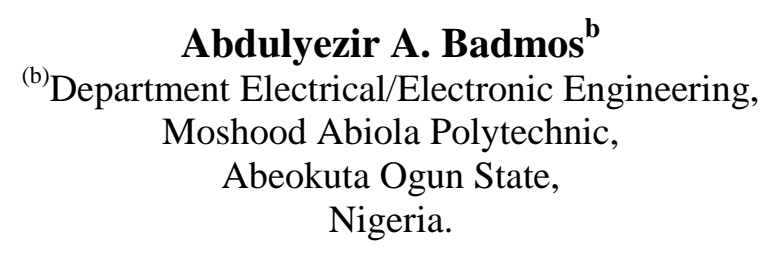

\author{
Musa A.I ${ }^{\mathbf{a}}$ \\ (a)Department Mechanical Engineering, \\ Moshood Abiola Polytechnic, \\ Abeokuta Ogun State, \\ Nigeria.
}

\section{Adeleke M.B ${ }^{\mathrm{a}}$}

(a)Department Mechanical Engineering, Moshood Abiola Polytechnic, Abeokuta Ogun State, Nigeria.
Abiodun O.I ${ }^{\mathrm{a}}$.
(a)Department Mechanical Engineering, Moshood Abiola Polytechnic, Abeokuta Ogun State, Nigeria.

Article DOI: https://doi.org/10.36713/epra6960 DOI No: 10.36713/epra6960

\footnotetext{
ABSTRACT

The word $3 D$ is defined as three dimensional models that display a picture or item in a form that appears to be physically present with a designated structure. Three-dimensional (3D), can also describe any object that occurs on a three-axis Cartesian coordinate system, a Cartesian system is basically a fancy way of describing the $X$ and $Y$ axes which are horizontal and vertical axis, but the inclusive of the third axis $Z$ which is make it to be $3 D$ of which the $Z$ axis represent the depth.3D interaction is a form of human-machine interaction where users are able to move and perform interaction in $3 D$ space. Both human and machine process information where the physical position of elements in $3 D$ space. The main features of these $3 D$ applications are immersion, interactivity, and involvement. Immersion holds the user's attention. Interactivity is related to how responsive the application is to user actions and Involvement has to do with engaging the user's interest in the underlying activity.3D interaction techniques are selection and manipulation, navigation, system control and symbolic inputs. Its application is in the area of Exploring Complex Data, Visualizations with the Cubic Mouse, Multimodal Interfaces in VEs Multimodal interaction, and VEs for Design Education Architectural design.

KEYWORDS:Interaction,cartesian,information,application,interface,virtual,physical,technique,immersion,involvement design, navigation and data.
} 


\subsection{INTRODUCTION \\ 1.1 WHAT IS 3D TECHNOLOGY?}

3D's. early beginnings can be traced back to 1962 when Morton Heilig invented the Senorama simulator. It provided 3D video feed back, but there is need to know the meaning of the word $3 \mathrm{D}$ ? . This is defined as three dimensional models that display a picture or item in a form that appears to be physically present with a designated structure. Essentially, it allows items that appeared flat to be display in a form that allows for various dimensions to be represented. These dimensions include width, depth and height. Three-dimensional (3D)also describe any object that occurs on a three-axis Cartesian coordinate system. According to Morton Heilig, the 3D feedback produces motion, audio, and haptic feedbacks to produce a virtual environment, the development was carried on by Dr.Ivan Sutherland,s of his pioneering work in 1968, he created a head-mounted display that produced a $3 \mathrm{D}$,

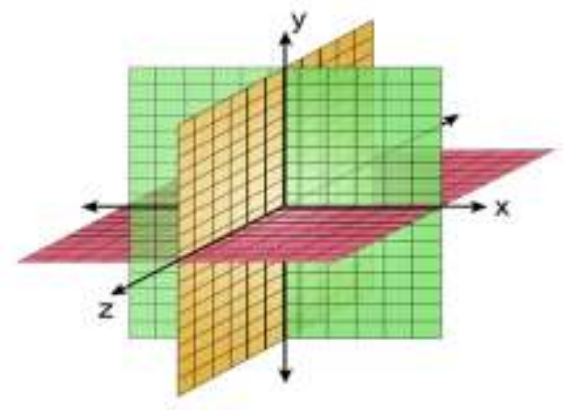

Figure 1 Shows the illustrations of 3D Cartesian graph and 3D image

3D Models: Any representation of an object in digital space is called a 3D model. If you take a look at the raw information that comprises a basic 3D model, it would simply (or not so simply) be a collection of data points that mark thousands or millions of different coordinates in Cartesian space more on the Z-axis since the $Z$-axis is such an essential characteristic of $3 \mathrm{D}$ space, let's take a closer look at what "Z" really means in a 3D software environment. The $\mathrm{Z}$ coordinate can be used to measure four things in 3D computer graphics:

1. The depth of an object in terms of size. As in, 5 units wide, 4 units tall, and 3 units deep.

2. The location of an object in relation to the origin. The origin in any $3 \mathrm{D}$ scene is $(0,0,0)$ with the third number usually being "Z". There are a few smaller 3D packages that use $\mathrm{Z}$ as the vertical axis, but these cases are rare.

3. The distance of an object from the rendered camera, known in computer graphics as $\mathrm{Z}$ depth. Z-Depth is often used to apply depth of field effects in post-production, and in video virtual environment by presenting a left and right still image of that environment.

.A three-dimensional graph is generally a graph of the function which denotes relationship among three variables. The graph of the function $\mathrm{f}(\mathrm{x}, \mathrm{y}, \mathrm{z})$ could be a three dimensional graph. A graph of $\mathrm{f}(\mathrm{x}, \mathrm{y}, \mathrm{z})$ can represent a curved or planar surface in the Cartesian coordinate system that is three dimensional. This graph is generally drawn on simply a two-dimensional screen or paper. It utilizes perspective ways in such a way that the third dimension seems to be seen on the screen.

The three dimensional Cartesian coordinate system is shown in the following diagram: It has $\mathrm{X}$ and $\mathrm{Y}$ coordinate axes which are mutually perpendicular, just similar to two-dimensional system and an additional third axis, called $\mathrm{z}$ axis that is also perpendicular to both $\mathrm{X}$ and $\mathrm{Y}$ axes. Have a look at a more clear three-dimensional image of three dimensional coordinate system.

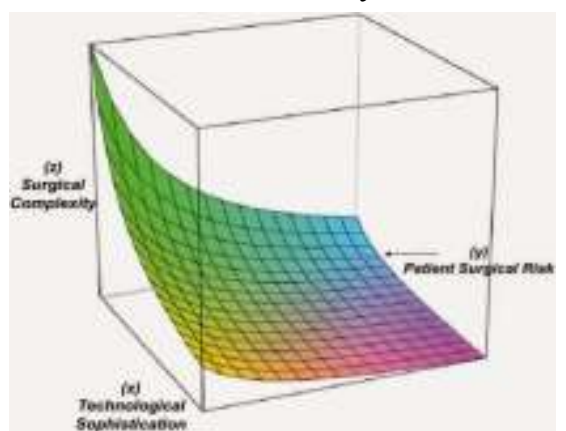

games it's used for level of detail optimizations.

4. The Z-axis of rotation. For example, a ball rolling away from a camera would be said to be rotating along negative of $\mathrm{Z}$-axis.

\section{Types of 3D Displays}

The most common form of 3D display is based on shutter technology. This is basically a form of alternating images by the display that are synchronized with some LCD glasses to alternate the two images between the perspective eyes. This technology is far from new and has been used with computers for many years through specialized hardware. The difference is that with fast LCD monitors and shutters, it is possible to produce these images in higher resolutions with greater refresh rates.Several efforts have been carried out to investigate the applicability of capitalizing on web-based architecture to run virtual application 


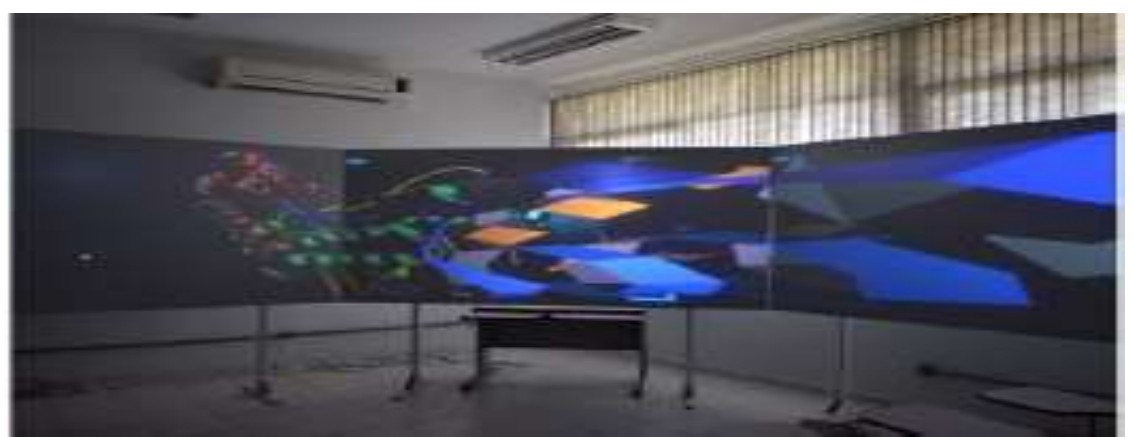

Figure 2 Shows visualization software that was ported to a mini-CAVE environment

\subsection{D INTERACTIONS}

In computing, 3D interaction is a form of human-machine interaction where users are able to move and perform interaction in 3D space. Both human and machine process information where the physical position of elements in 3D space is relevant, it means users carry out their tasks and perform functions by exchanging information with computer systems in 3D. The 3D space used for interaction can be the real physical space, a virtual representation simulated in the computer, or a combination of both. When the real space is used for data input, humans performs actions or give commands to the machine using an input device that detects the 3D position of the human action. According to virtual reality applications allow users to take part in experiences that are either very difficult or impossible to take place in real life (Herndon, van Dam, \& Gleicher, 1994)for instance, a microscopic world, a distant planet, a fantasy world, an expedition inside an erupting volcano. Through these applications, users are able to enter a virtual environment that can be manipulated to varying degrees and explored in real time. To allow for this interaction, these applications use special-purpose devices that engage the basic senses (e.g., vision, hearing, and touch). Therefore, due to their potential to complement and augment the learning process, these applications are being increasingly deployed in many contexts (Abbasi A etal 2012), (Finkelstein S etal 2011), ( Li M, Buchthal S (2012)),(Lv Z.etal 2016),(Muhanna MA (2015), (Pedras BFV. etal 2013), for example, Mathematics, Chemistry, Flight Simulators, Sales and Marketing, Demo Prosthesis (Medical)Real time interactive application (RIA)and Geographical Information Systems. The main features of these 3D applications are immersion, interactivity, and involvement. Immersion holds the user's attention. Many immersive technologies can be used to achieve high levels of immersion as, for instance, 3D stereoscopic displays and motion tracking devices. Interactivity is related to how responsive the application is to user actions. Involvement has to do with engaging the user's interest in the underlying activity and it can be achieved by providing engaging challenges to the user. In the early years, 3D applications required substantial computing power, thus high-end supercomputers were needed (Cruz-Neira. etal 1992). Development of these applications was further complicated due to the lowlevel software platforms available at the time. In recent years, however, given the advances in computer hardware, software, and network, these applications have become more affordable.3D is also used in the development of human robot interaction technologies, haptic interfaces to provide the sense of touch. These interfaces have been utilized in medical simulation, virtual assembly and remote manipulation tasks. It is also used for teaching guidance, navigation, and control concepts of a quadrotor to undergraduate students, using a graphical user interface (GUI) and 3D animations. In the past seventeen years, 3D (VD) application has witnessed a significant transformation in nature and sophistication since the introduction of visual interactive simulation/modeling. 3D interfaces have been used in applications that feature virtual environments, and augmented and mixed realities. In virtual environments, users may interact directly with the environment or use tools with specific functionalities to do so. Therefore, 3D interaction occurs when physical tools are controlled in 3D spatial context to control a corresponding virtual tool. Users experience a sense of presence when engage in an immersive virtual world as mentioned above, this enable the users to interact with this world in $3 \mathrm{D}$, it allows them to make use of natural and intrinsic knowledge of how information exchange takes place with physical object in real world. The users must have a way of performing actions in 3D as well, therefore special input and output devices to support this type of interaction such as 3D mouse which is developed based on existing devices for 2D interaction. Therefore, great 
care must go into the design of user interfaces and interaction techniques for 3D applications Schaeffer B. Gouseune C (2003). All interactions in the virtual training environment could be equally carried out using either hand and participants could concurrently complete one interaction with each hand. For example, a participant could grab and rotate the assembled pieces with one hand and grab the next block to attach with the other hand
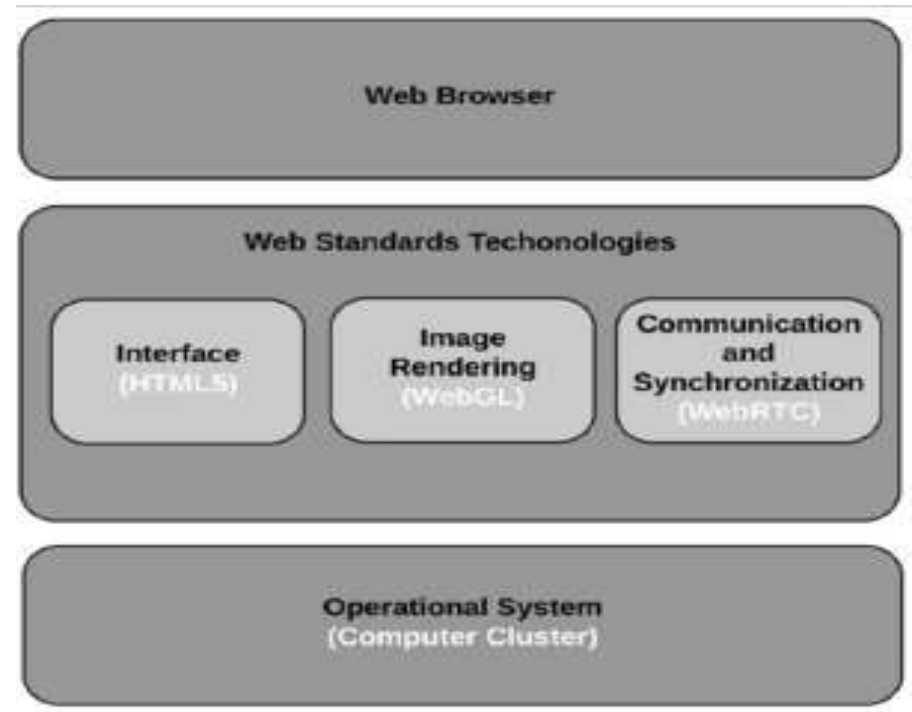

Figure 3: Software architecture to develop immersive and interactive applications to run web browsers

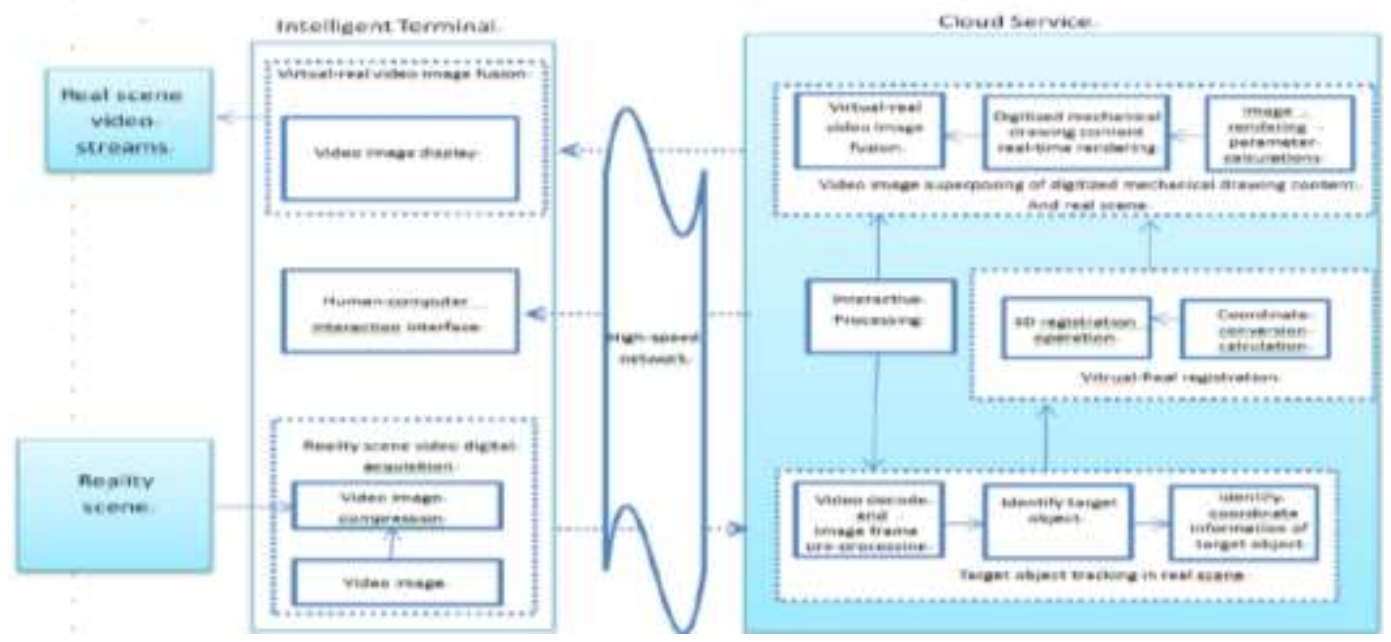

Figure 4: Functional diagram showing Mechanical interactive Teaching System

Case Study in Figure.3 illustrating an architecture porting an HTML5 application to run in immersive and interactive environment.
The diagram in Figure.4 illustrate the teaching system based on the Unity 3D game engine (Unity3D:https://unity3d.com/cn/), Qualcomm Vuforia and uses $3 \mathrm{ds}$ max for 3D digital models. The functional 
diagram of the mechanical drawing interactive teaching system is based on the augumented reality approach as design in this paper.

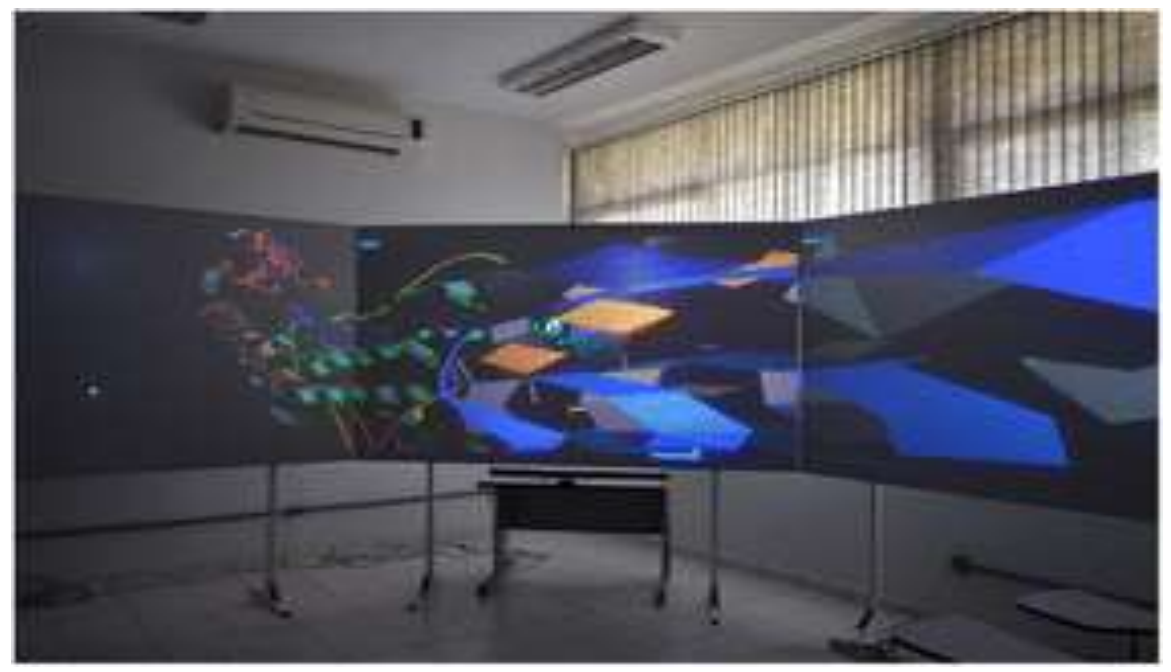

Figure 5 Visualization software that was ported to a mini-CAVE environment

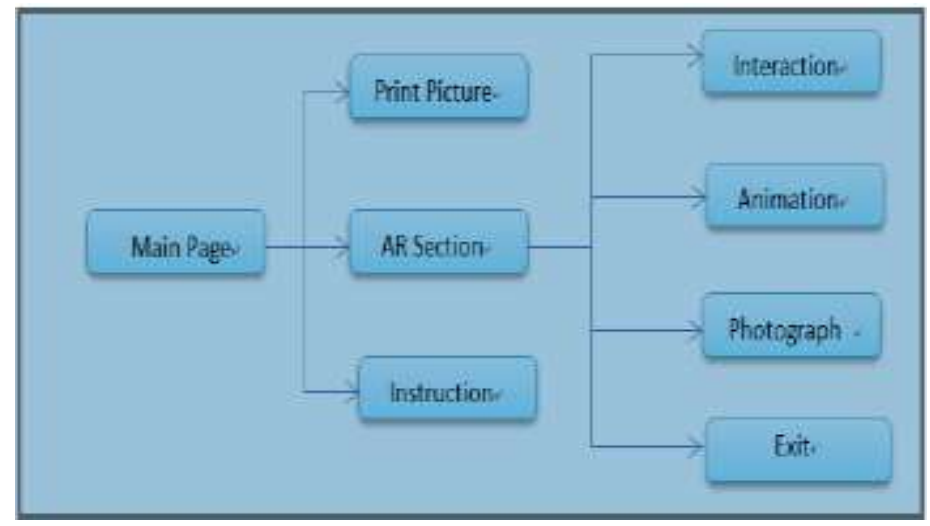

Figure. 6 Functional diagram of Mechanical Drawing interactive teaching system

\subsection{D USER INTERFACES}

User interfaces are the means for communication between users and systems. This includes media for 3D representation of system state, and media for 3D user input or manipulation. 


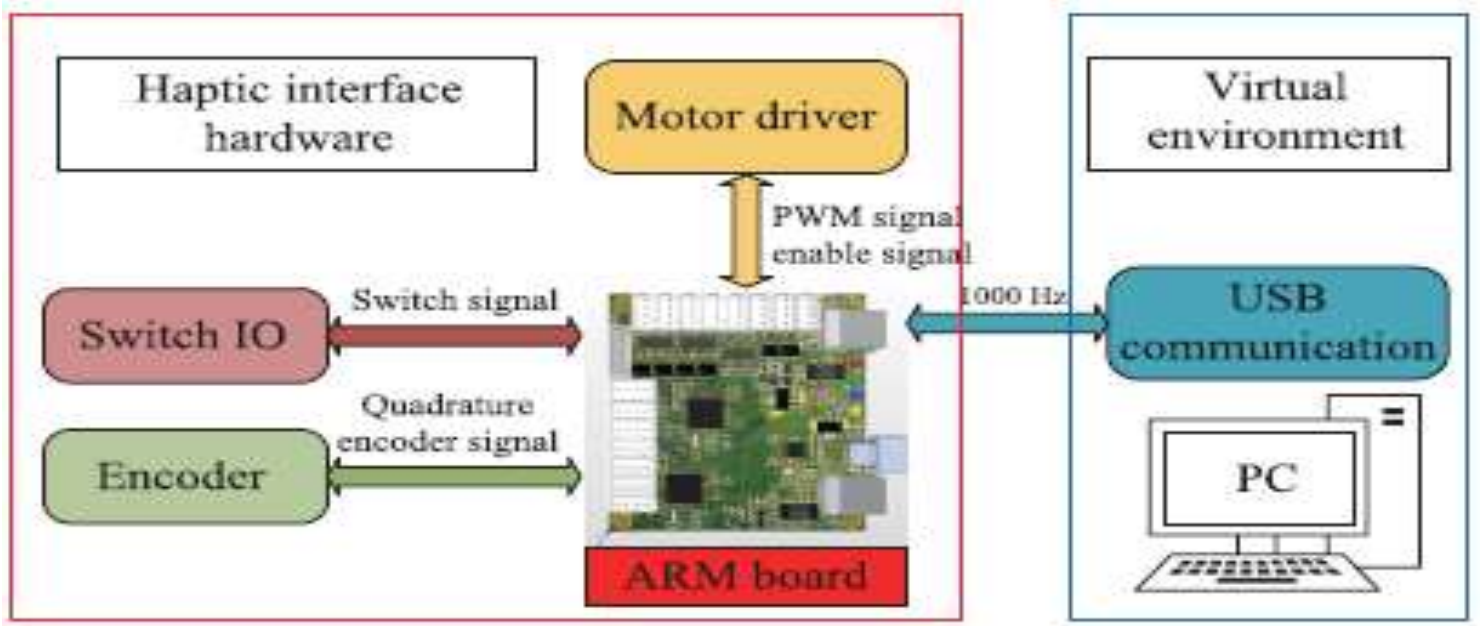

Figure 7Interface between Virtual environment and USB Communication System

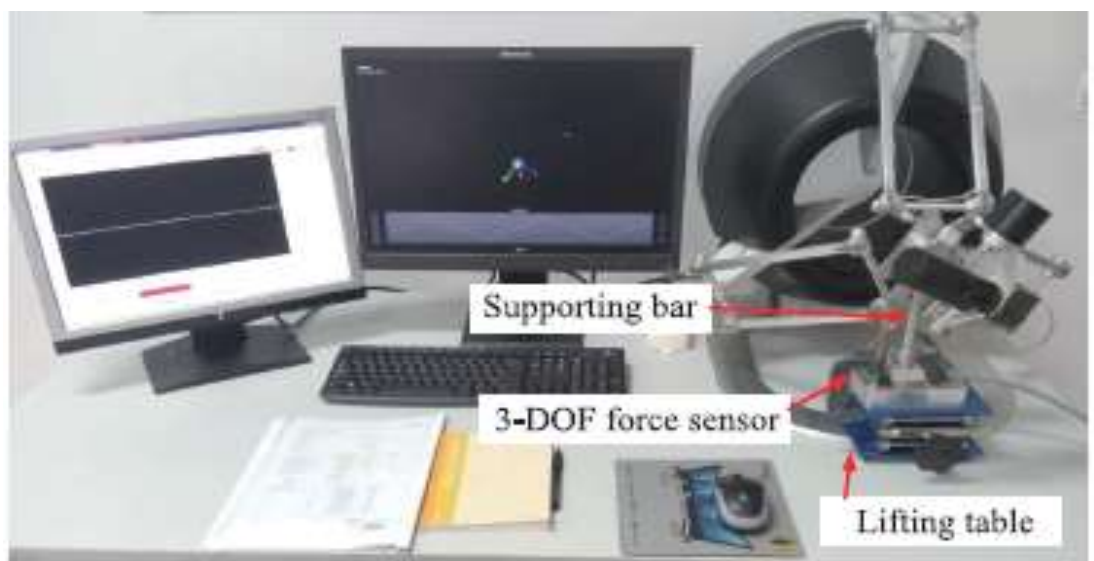

Figure 8.An experiment set up of Haptic interface between the System and the virtual environment

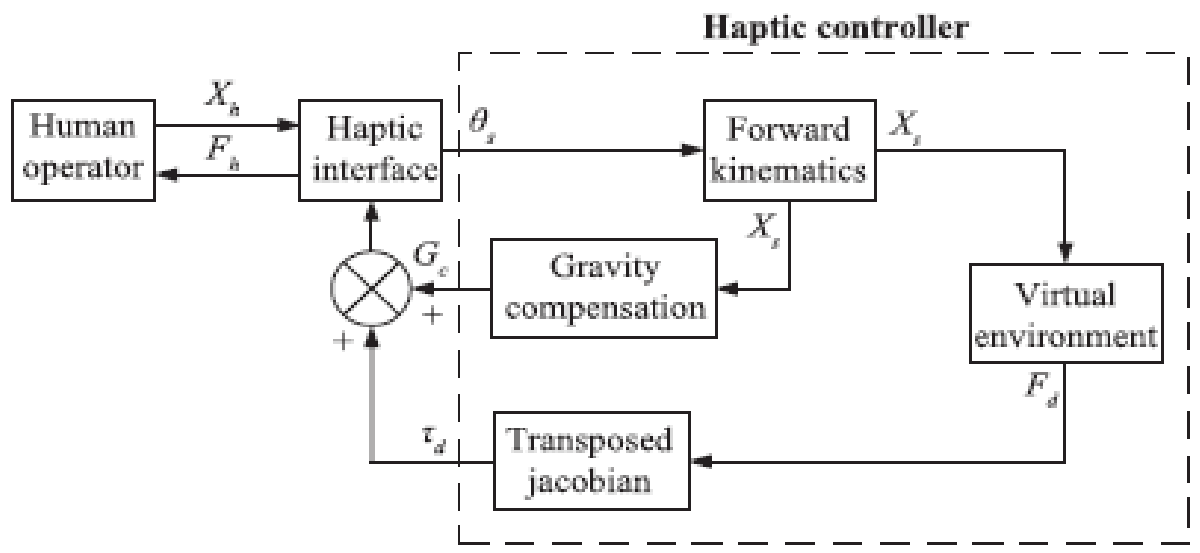

Figure 9 Block diagram showing control called “Open Loop” due to no feedback from output Controller 


\subsection{Input devices}

These input devices are instruments used to manipulate objects and send control instructions to the computer system. A distinction must be made between input devices and interaction techniques. Input devices are just the physical tools used to implement various interaction techniques. Input devices can be roughly categorized based on the types of events they generate, Devices that generate one event at a time based on the user are considered discrete-input devices example is a Pinch gloves, developed by Fakespace, is an example of a discrete-input device (the user pinches two or more fingers to signal an event). In contrast to discrete-input devices, continuous-input devices generate a stream of
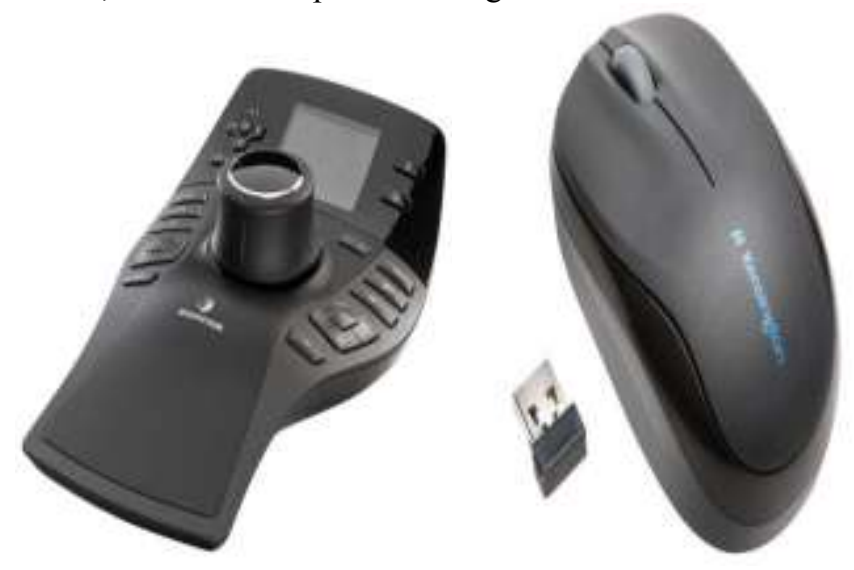

Figure 10 Shows examples of input devices

events. Two of the most common continuous-input devices are trackers, control devices, navigation equipment and gestures interfaces. Standard input devices include keyboards, tablets and stylus, joysticks, mice, touch screens, knobs, and trackballs. Devices that combine both discrete and continuous events to form single, more-flexible devices are called combination or hybrid-input devices. Examples of hybrid devices include the Ring Mouse (a small device worn on the user's finger that combines ultrasonic tracking with two buttons) and pen-based tablets, which are becoming more popular in VE applications because they give users the ability to interact in two dimensions.

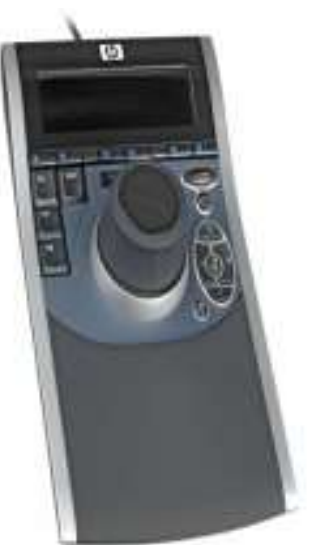

\subsection{Output devices}

Output devices allow the machine to provide information or feedback to the user, but we commonly use the term display to describe output. Although the most common displays are visual, there are other important displays which include auditory, haptic, tactile, and olfactory channels. Visual displays provide feedback to users in 3D visual form. In the context of 3D user interfaces for VEs, visual displays can be roughly categorized into fully immersive and semiimmersive devices. Fully immersive displays - such as head mounted displays (HMDs), virtual reality headsets, cave automatic virtual environment (CAVEs), armmounted displays, and virtual retinal displays - occlude the real world as shown in Fig.11below. Semiimmersive displays such as stereo monitors, workbenches, and surround-screen virtual reality systems allow the user to see both the physical and virtual world. This is especially useful when supplying location and spatial information to the users, addition of background audio component to a display adds to the sense of realm. Haptic displays send tactile feedback to the user (Burdea, 1996). 


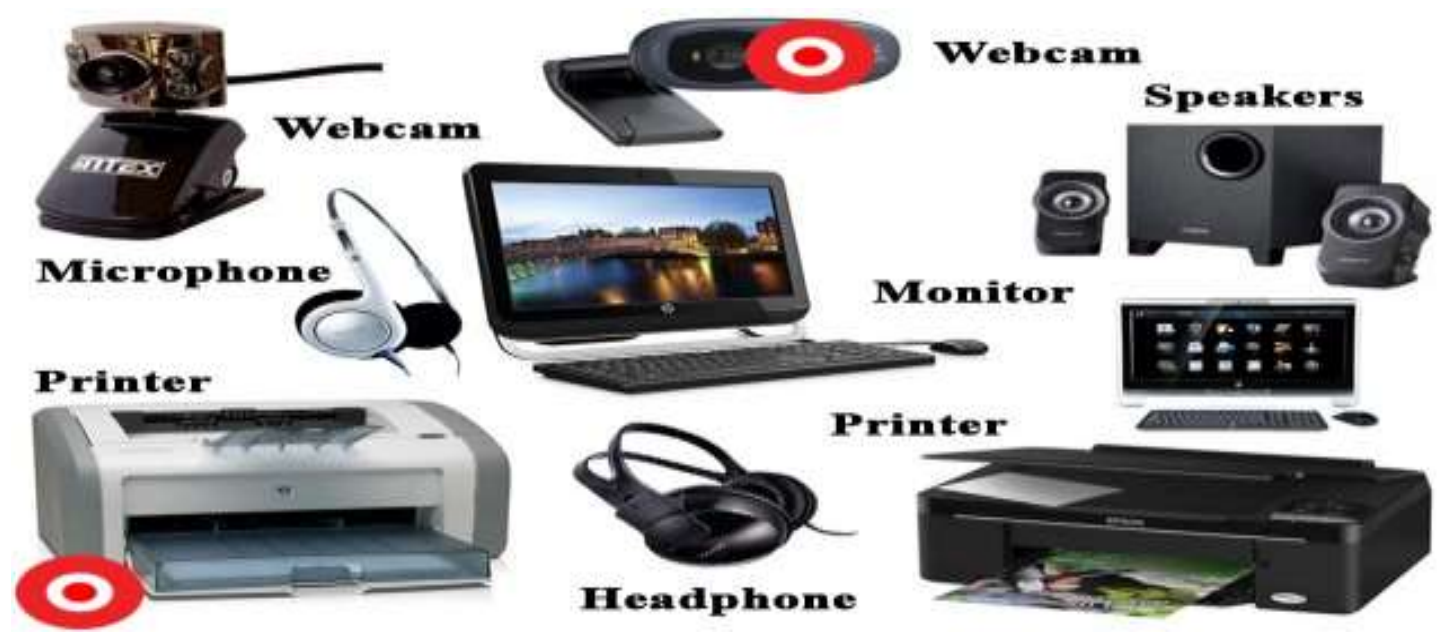

Figure 11 shows an examples of 3D output device

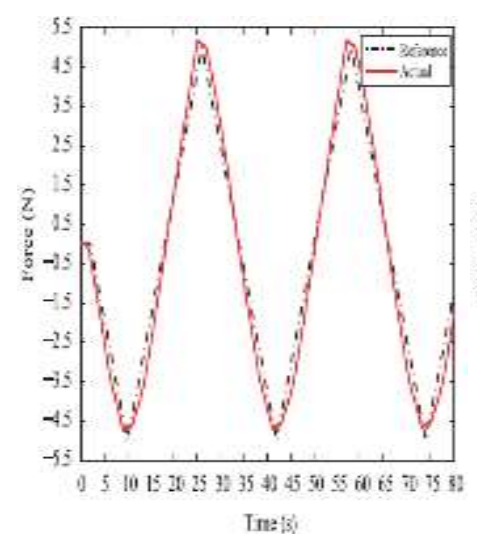

(a)

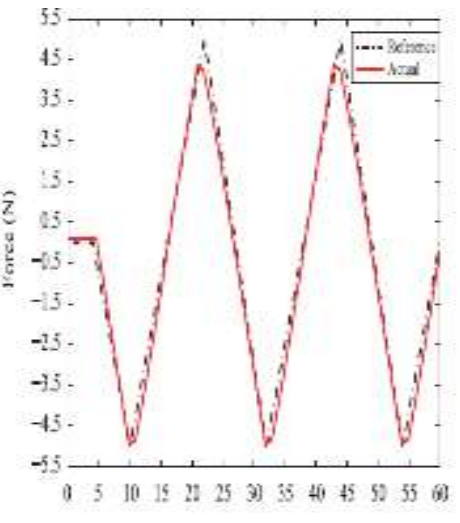

(b)

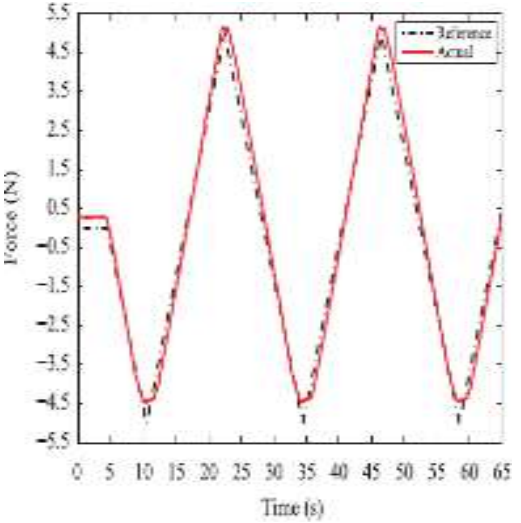

(c)

Figure 12 Graph showing example of Force Output Haptic Interface along 3(D) axes $(x, y, z)$ with Time in an Experiment

\subsubsection{D Interaction Techniques}

3D interaction techniques are methods used in order to execute different types of task in 3D space. Techniques are classified according to the task they support.

\subsubsection{Selection and Manipulation}

Interaction techniques for 3D manipulation in VEs should provide means to accomplish at least one of three basic tasks: object selection, object positioning, and object rotation. This is because users need to be able to manipulate virtual objects. Manipulation tasks involve selecting and moving objects, sometimes, rotation of the objects is involved as well. Direct hand manipulations are the most natural technique because manipulating physical objects with the hand is intuitive for humans. However, this is not often possible, the classical approach to design manipulation techniques is to provide the user with a "virtual" hand, a 3D cursor, often shaped like a human hand, whose movements correspond to the movements of the hand tracker. Selection and manipulation ply involve touching an object, then positioning and orienting this virtual hand within the VE.3D widgets can be used to put controls on objects: these are usually called 3D Gizmos or Manipulators (Blender 1998).Users can employ these to relocate, re-scale or re-orient an object. Other techniques include the Go-Go techniques (Poupyrev, Billinghurst, Weghorst, \& Ichikawa, 1996) and ray casting where a virtual ray is use to point to and select an object, the extension of the user's reach by using a nonlinear mapping applied to the user's hand extension. When the user extends the hand farther than a threshold distance $\mathrm{D}$, the mapping becomes nonlinear and the virtual arm "grows. Different mapping functions can be 
used to achieve different control-display gain between real and virtual hands (Bowman \& Hodges, 1997).

\subsubsection{Navigation}

The computer needs to provide the user with information regarding location and movement. Navigation tasks have two components.

Way-finding: refers to finding and setting routes to get to a travel goal within the virtual environment, studies with maps (Darken \& Cevik, 1999).

Travel:Good travel techniques allow the user to easily move through the environment.There are three types task namely; exploration, search, and manoeuvring techniques. It can also be classified into the following:

- Physical movement- user moves through the virtual word

- Manual Viewpoint manipulation-use hand motions to achieve movement

- $\quad$ Steering- direction specification

- Target-based travel- destination specification

- Route planning-path specification

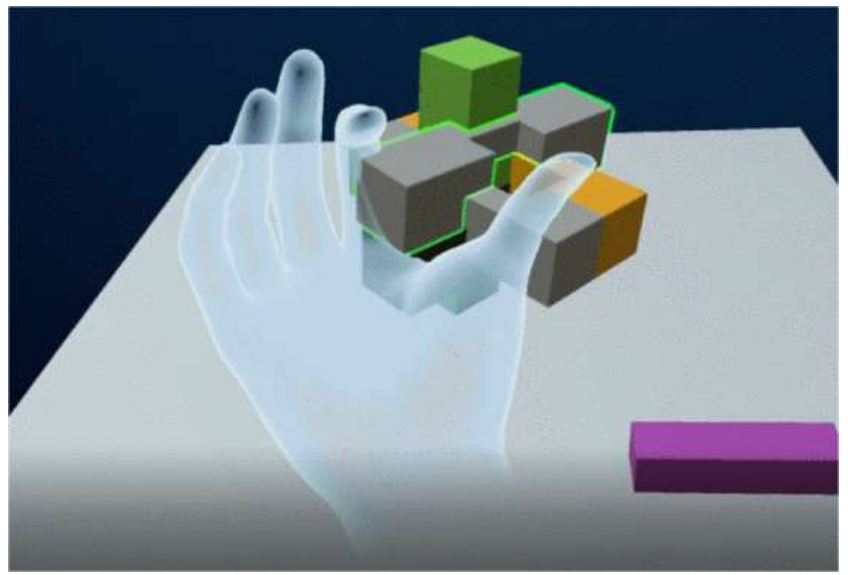

Figure 13 Screenshot of a participant grabbing a virtual block and assembling it onto the 3D puzzle. The twenty degrees from the correct orientation

\subsubsection{System Control}

Tasks that involve issuing commands to the application in order to change system mode or activate some functionality fall under the category of system control. Techniques that support system control tasks in threedimensions are classified as:

- Graphical menus

- Voice commands

- Gestural interaction

- Virtual tools with specific functions

\subsubsection{Symbolic Input}

This task allows the user to enter and /or edit, for example text, making it possible to annotate $3 \mathrm{D}$ scenes or 3D objects.

\subsection{D INTERACTIVE APPLICATIONS 4.1.03 $D$ interactive Application for Education}

3D Interactive Learning Technologies provides interactive learning tools for training purposes. It is a package of tools that can be tailored to any learning criteria. It is possible to create a tailor made system for any real world training scenario. It is also use in game developing; developers have battled each other for decades about whether voxels or polygons are best for game graphics. Atomontage'sfounders believe voxels are a much better way to create fully interactive 3D applications, and they're unveiling a new platform to enable voxel art in high-end video games and other 3D applications. The technology will enable volumetric simulations to scale massively in fully interactive 3D applications, with billions of voxels."The currently dominant 3D graphics and simulation paradigm, based on polygon meshes, this show that augmented reality and virtual reality rendering and interaction with large voxel scenes is possible on low-end VR-ready hardware today as shown in fig.14below.Data visualization techniques are also a new method which provides new methods for the generation of interactive graphs(R.R. Dev. Team Core 2011). These graphs allow a better exploration and interpretation of data but their creation requires advanced knowledge of graphical libraries as shown in fig. 14 and fig.15below. 

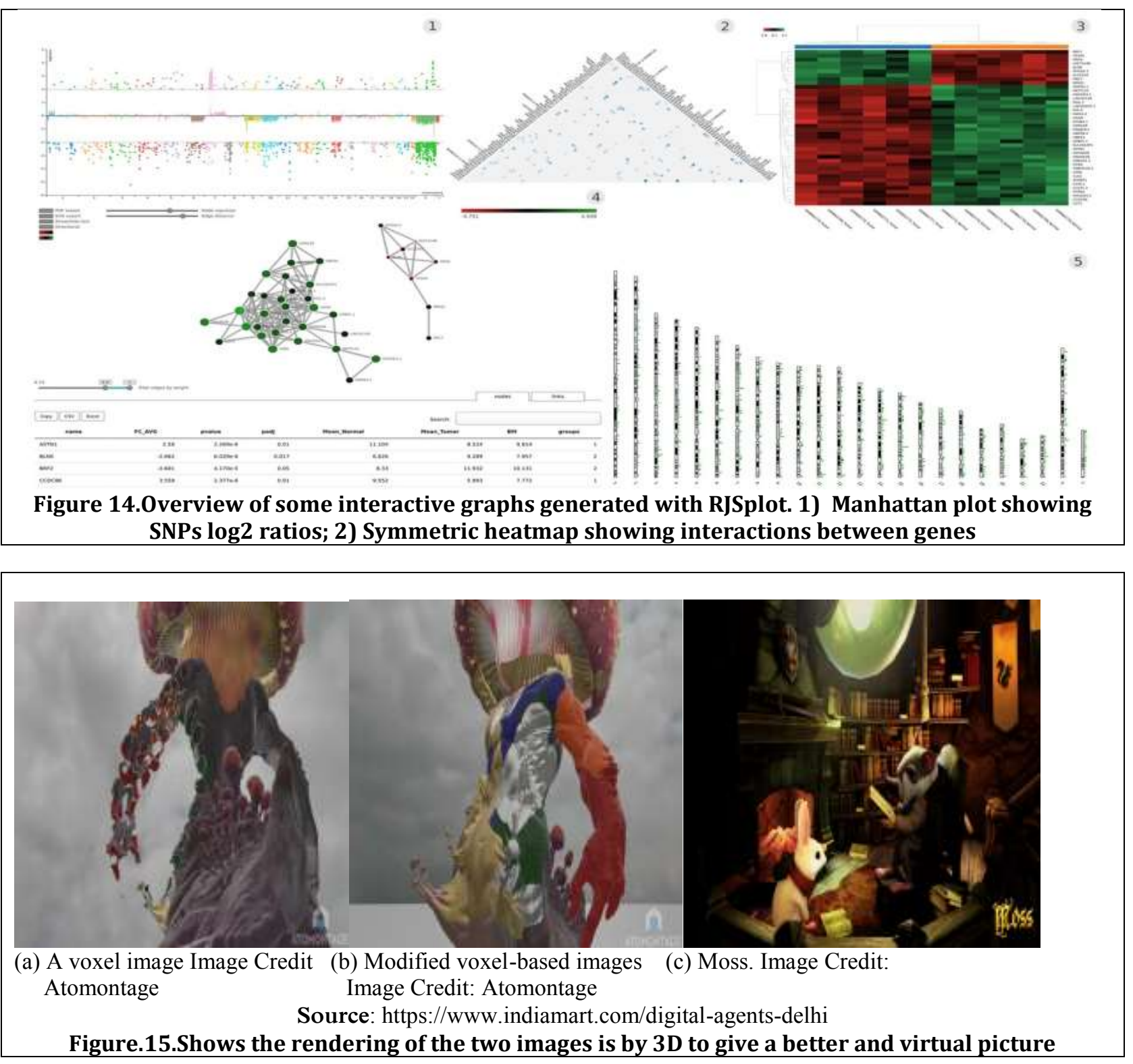

\subsubsection{Applications in the Area of Medical 3D Anatomy for human learning}

Medical professionals have always been trained using human anatomy models. Earlier these anatomy models were made from wax. Later on industrially manufactured plastics replaced these models. Modern information and visualization using 3D technology offers a real alternative to anatomical plastics models, it provides a visual presentation that disseminates scientific, medical and biological knowledge with highly detailed anatomical cross-sections, it conveys medical, biological, scientific, and other life science topics visually. They improve the impact and knowledge transfer of research publications.3D human anatomy images give you detailed 3D models of all major organs and systems of the human body. With easy to use 3D controls and dynamical search capability, these applications are usually free online. 3D human anatomy atlas is not only used by med. students, teachers, but anyone who wants to learn more about the human body. The models are developed using novel 3D visualization techniquesby (Soffers.etal (2015)) with decades of experience in the fields of medical illustration and biomedical visualization behind them, example is shown in the fig 16below. 

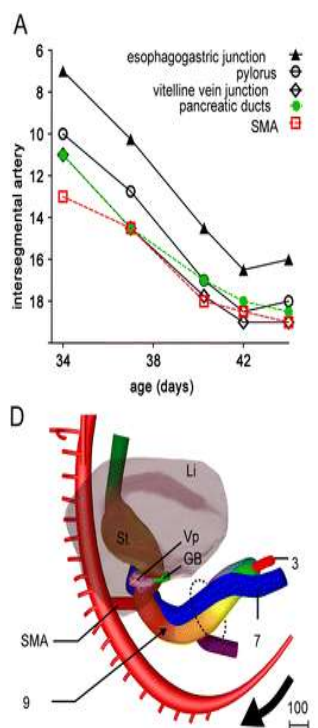
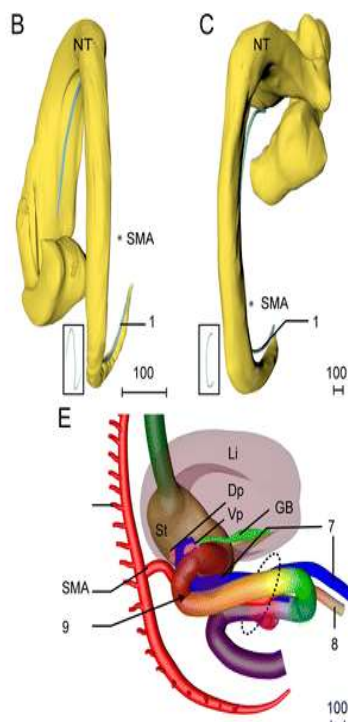

(a)

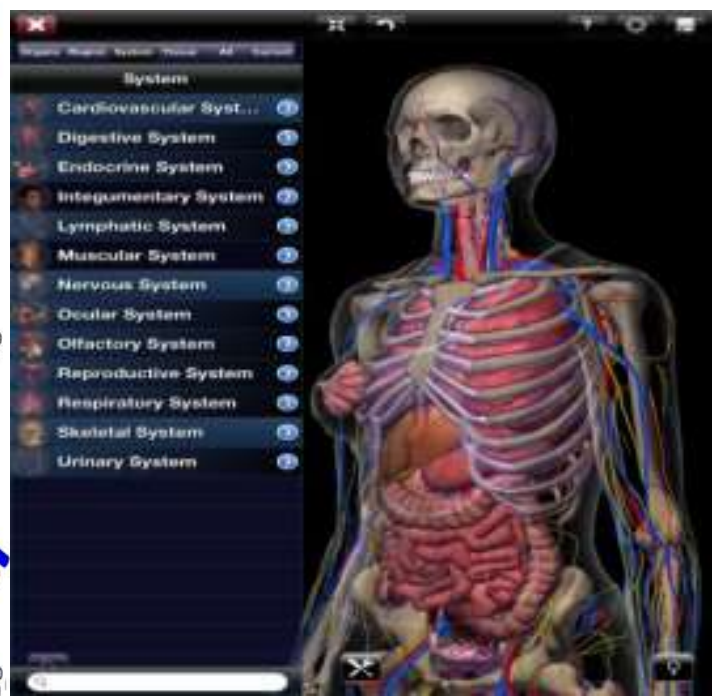

(b)

Figure 16. Shows 3D technique application for human anatomy.

IIITD student develops app for 3D, interactive viewing of CT/MRI scans named "RealVol," which will help view CT/MRI images in a virtual reality environment with three-dimensional perception. (Press Trust of India 2018). A new virtual reality (VR) system, developed in collaboration with trained neuroanatomists, to trace neurons in microscope scans of the visual cortex of primates using consumer-grade VR technology to interact with neurons directly in 3D will help neuroscientists better resolve complex cases and enable them to trace neurons faster and with less physical and mental strain (Will Usher .etal.2018).This is shown in fig. 17 below.

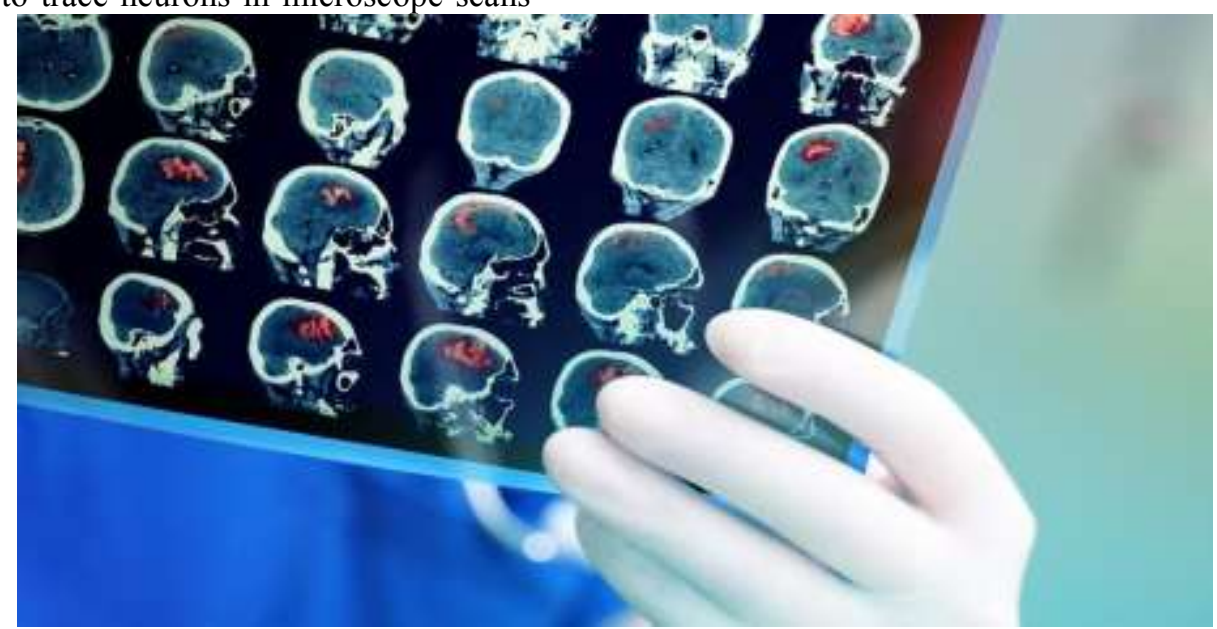

Figure 17:3D, interactive viewing of CT/MRI scans named "RealVol," which will help view CT/MRI images in a virtual reality environment

4.1.23D Techniques Application in Automotive and Transportation Industry (creaform)

Creaform is a key partner in the automotive and transport industries, 3D measurement technologies can be used for applications such as:
- Styling, design and reverse engineering

- Prototyping

- Simulation

- Manufacturing

- Quality control/inspection

- Design for aftermarket 
An example of 3D creaform used in automotive design is seen in Fig.18below

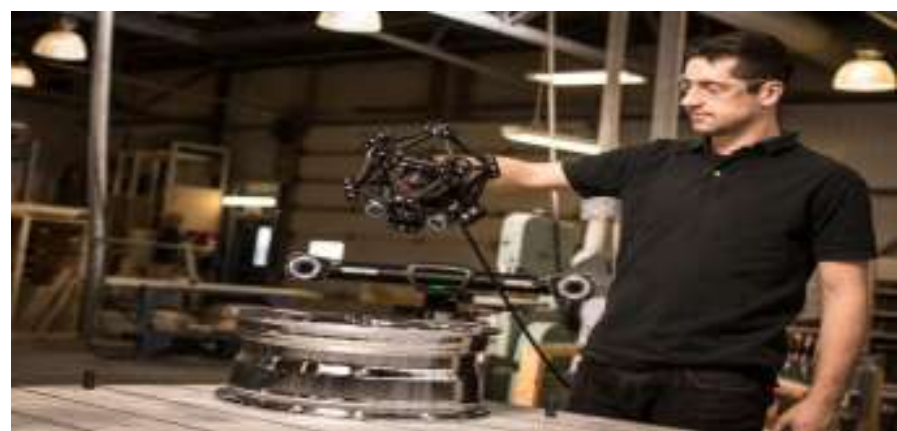

Figure 18 Shows cream forming of car alloy wheel in the automobile manufacturing shop

\subsubsection{D Interactive application in printing (3D)}

$3 \mathrm{D}$ printing is a process in
which material joined or solidified under computer control to create a three dimensional object, with material be in gadded together.3D printing is used in both rapid prototyping and additive manufacturing. Current industrial 3D printers are recognized for usefulness in many companies with high-quality outputs. The use printers (3D) in the automotive design field at the moment are the state stopping to sample production or prototyping technology public relations degree level. 3D enhances effect on the Transportation Design and Printing Process by an analysis of the information and this technology and help to be actively utilized.

Objects can be of almost any shape or geometry and typically are produced using digital model data from a 3D model.(J.of Korean Society of Design Culture (2017)). The example of 3D printing is seen in Fig.19below showing the drawing and printing (rendering) of motorcycle.

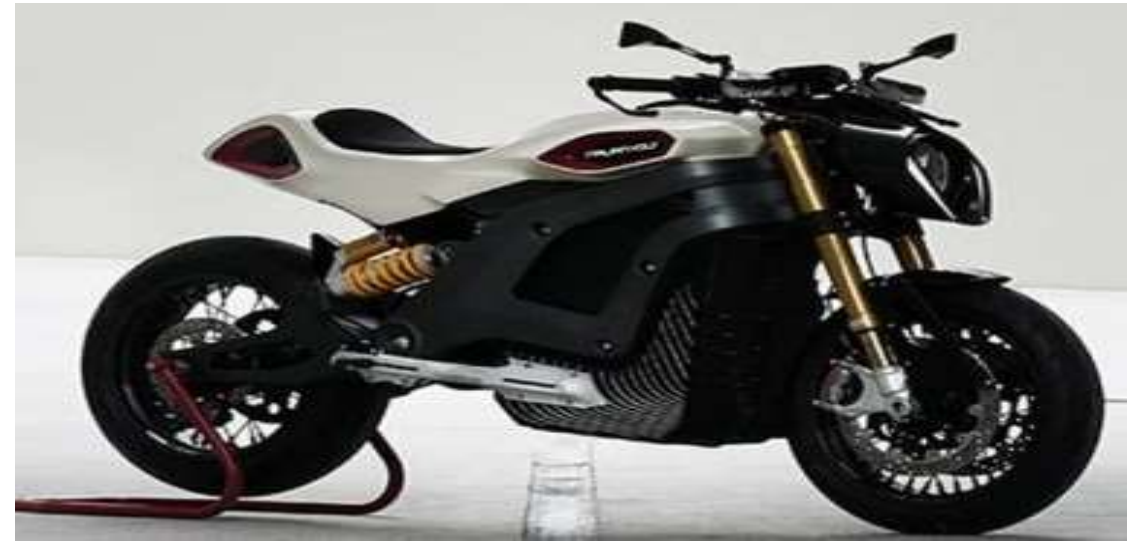

Figure 19 shows 3D Printing (rendering) of a motor bike

Source: https://3d printing industry.com/news/best-aerospace-automotive-3d-printing-applications-2018-132091

\subsubsection{D Interactive Applications on Sales and Marketing}

Big Data is the biggest hame-changing opportunity for marketing and sales since the Internet went mainstream almost 20 years ago. The data big bang has unleashed torrents of terabytes about everything from customer behaviors to weather patterns to demographic consumer shifts in emerging markets. The companies who are successful in turning data into above-market growth will excel at three things:

- Using analytics to identify valuable business opportunities from the data to drive decisions and improve marketing return on investment (MROI).

- Turning those insights into well-designed products and offers that delight customers. 
- Delivering those products and offers effectively to the marketplace.

This goldmine of data represents a pivotpointmoment for marketing and sales
leaders.Companies that inject big data and analytics into their operation show productivity ratesand high profitability. This is shown in Figure 20 below.

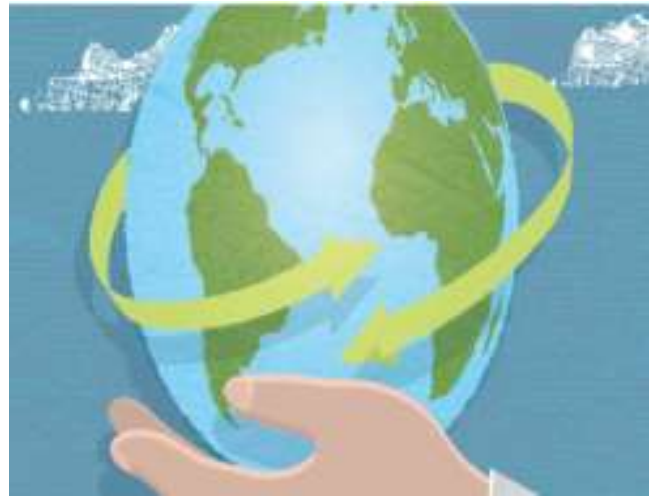

(a)

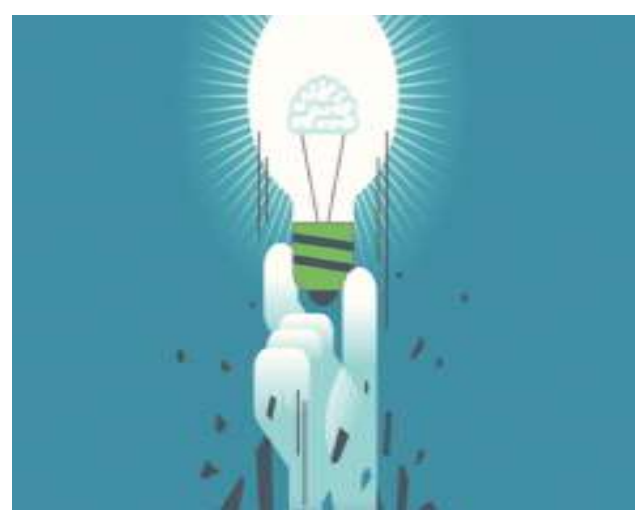

(b)

Figure 20 Shows how to get big impact on sales through big data in the micro market (Marketing and Sales across the world) Source: htts://www.kaon.com

\subsubsection{Interactive Plant Simulation Modeling to Avert Industrial Accident}

Model structure The interactive plant simulation model interlinks process and accident simulation models in an overall training scenario from process upsets to accident occurrence and propagation In this model, three simulations are linked based on a certain sequence of accident scenarios: dynamic process simulation, discharge calculation, and pre-calculated 3D-CFD simulation. Through this simulation linking structure, the model leads trainees to actively analyze process variable trends based on the simulation results and take proper actions with their own decisions to stabilize the variables or minimize operational losses and accidents. When stabilization fails and an accident occurs, associated results like gas cloud concentration and explosion overpressure at each time and position are additionally provided to the trainees. Their actions like emergency shutdown can be inputted by clicking a mouse or a joystick control, the process is shown in Fig. 21 below

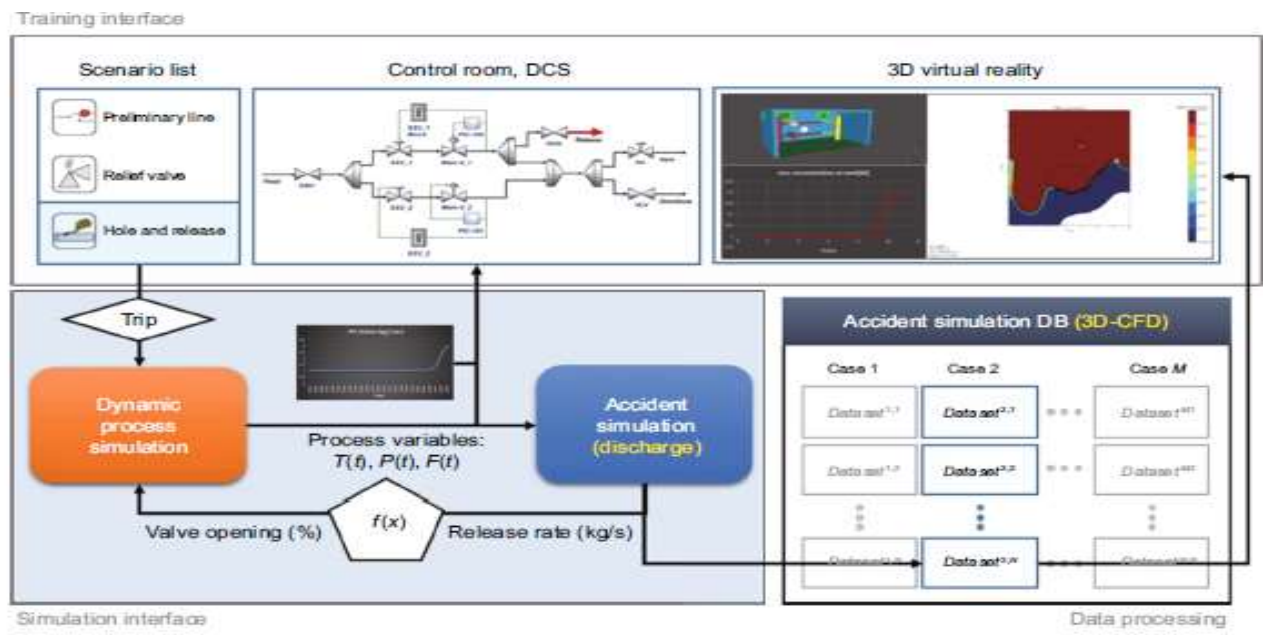

Figure 21 Shows Interactive Simulation Model for Industrial accident Prevention 
This involves the PFD status of controller alarms such as high or low alarms when the pressure goes beyond the set limit. The process upsets and accident scenarios on the left list were set up to initiate the desired scenario by clicking the button for a relief, the fig.22below shows

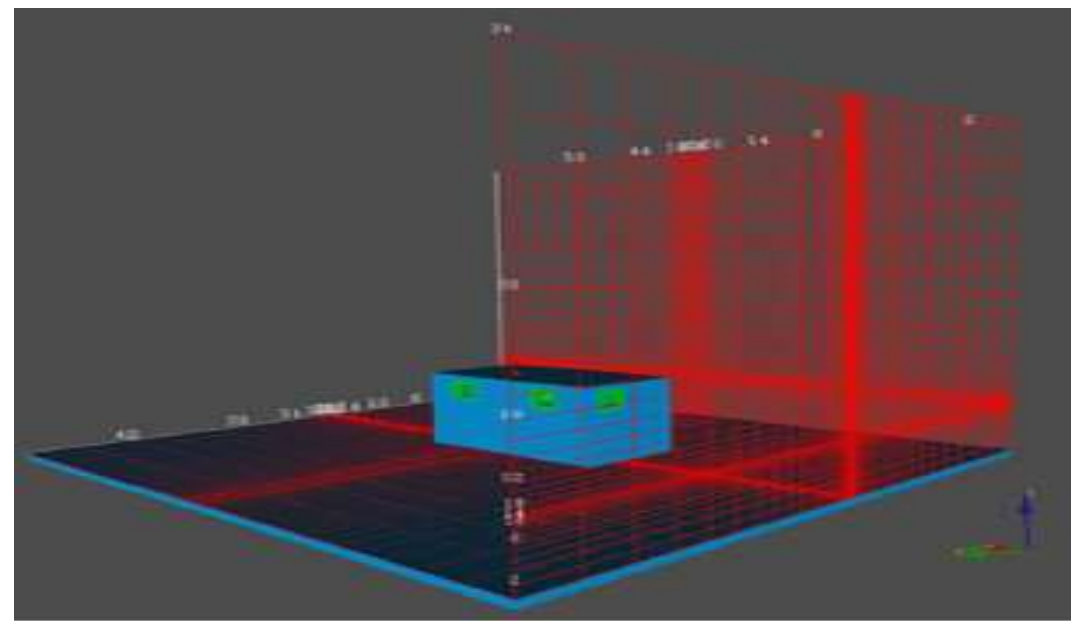

Figure 22: Accident simulation 3D-CFD using FLACS in gas industry

\subsubsection{D Application in Entertainment Industry}

Use of 3D Technology in industry is evident everywhere. Whether it is entertainment, games or movies, companies large and small keep on looking for ways to squeeze efficiencies out of 3D technology today. This technology seems to penetrate all realms of the world today. Quite recently; the 3D's advertisement
Propaganda is everywhere. People want to try and see something new.3D technology in industry can already be seen impacting the home market. 3DTVs and 3D Blu-ray players are still very new, but significant developments have been made recently. This time too, use of 3D Technology in industry will sure take the advantage of the latest technological development in boosting its market as shown in fig. 23 below.
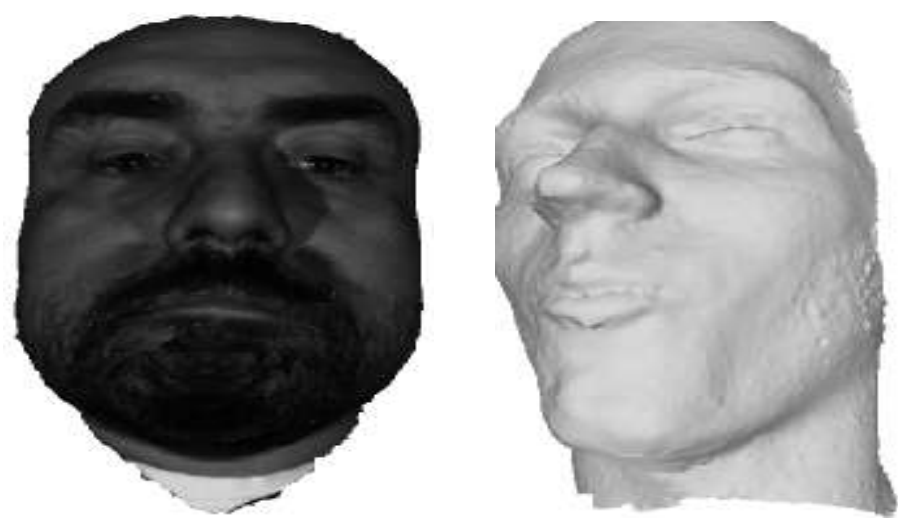

Figure 23 : Shows Preprocessing of 3D Scanned Images for Facial Animation on the basis of Realistic Aquisition

\subsubsection{Multimodal Interfaces in VEs Multimodal interaction}

This can be defined as the combination of multiple input and output modalities Bowman et al. It provide users with a richer set of interactions compared to traditional unimodal interfaces. Output modalities can be combined to allow for sensory substitution and parallel processing of information. There are several basic ways to combine input modalities (Martin, 1998), including complementarity (two or more input modalities complement each other when they combine to issue a single command), concurrency (two or more input modalities are concurrent when they issue different commands that overlap in time), and transfer 
(two input modalities transfer information when one receives information from another and uses this information to complete a given task).

\subsubsection{VEs for Design Education Architectural design}

This is often touted as an application area for which virtual environments should be ideal. Beyond walkthroughs and validation environments, however, there are few applications of immersive design. The Virtual Habitat (Bowman et al., 1998) is a constrained design space focused on education, rather than a generic design environment aimed at professionals. The goal of the Virtual Habitat is to allow students to apply the principles of environmental design. The task of immersive design is made possible because students do not have to start with a blank environment and because the design tools are constrained by the specific tasks of exhibit design and by the principles of good environmental design.

\section{Exploring Complex Data Visualizations with the Cubic Mouse}

The use of complex visualization systems is widespread in application domains such as the automobile industry, the medical field, and the oil and gas industry. These visualization systems need to handle massive amounts of data, use appropriate visualization methods, and apply effective interaction techniques to inspect and manipulate data. Traditional input devices often form a bottleneck when interacting with such systems, due to their imprecision and need for regular mode changes. At the German National Research Center for Information Technology (GMD), research in visualization has led to a new input device called the Cubic Mouse (Froehlich \& Plate, 2000).

\subsection{D INTERACTIONS DISPLAY LIMITATIONS}

- Even with all of the various 3D technologies, there is a segment of the population that lacks the physical ability required to see the images properly. For some this means they will still just see a two dimensional image while it can induce headaches or disorientation in others. In fact, some manufacturers of the 3D displays are putting warnings on their products to suggest against extended use due to these effects.

- The next problem is the fact that you will need to have specific hardware in order to use it. In the case of the glasses technology, you have to have a display and a compatible pair of shutter glasses in order to use it. This isn't too much a problem on a single user environment like a computer but is more problematic with a standard TV where multiple users would each need a pair of compatible glasses.

- The other problem is that glasses for use with one monitor may be inverted from another presenting the wrong image to the wrong eye. There are major limitations to this technology including the need to greater space for the display, lack of color and their high costs.

\subsection{CONCLUSIONS}

Three-dimensional interaction application and research will only continue to grow in importance as VEs become more complex and widespread. Several generic principles for $3 \mathrm{D}$ user interface design can be stated based on the current state of the art in this area. These include:

- Consider "magic" interfaces in place of "natural" ones when tasks require productivity and efficiency. Natural interaction should be used when a replication of the physical world.

- Choose interaction techniques based on the requirements of the application. The same set of techniques will not work well in every situation.

- Limit the required degrees of freedom for input whenever possible, and provide physical or virtual constraints to help guide user input.

- Take advantage of the increased flexibility afforded by whole-body input, multimodal I/O, and novel input devices

\section{REFERENCE}

1. Abbasi A, Baroudi U (2012) Immersive environment: an emerging future of telecommunications. IEEE MultiMed 19(1):80. doi:10.1109/MMUL.2012.7.

2. Finkelstein S, Suma EA (2011) Astrojumper: motivating exercise with an immersive virtual reality game.Presence:Teleoper.Virtual Environ 20(1):78-92. doi:10.1162/pres_a_00036.

3. Li M, Buchthal $S$ (2012) Advisory services in the virtual world: an empowerment perspective 12(1), 53-96. doi: 10.1007/s10660-012-9088-6.

4. Lv Z, Li X, Zhang B, Wang W, Zhu Y, Hu J, Feng S (2016) Managing big city information based on webvrgis. IEEE Access 4:407-415. doi:10.1109/ACCESS.2016.2517076.

5. Lv Z, Yin T, Song H, Chen $G$ (2016) Virtual reality smart city based on webvrgis. IEEE Internet Things J PP(99):1. doi:10.1109/JIOT.2016.2546307.

6. Muhanna MA (2015) Virtual reality and the cave. J King Saud Univ Comput Inf Sci 27(3):344-361. doi:10.1016/j.jksuci.2014.03.023. 
7. Pedras BFV, Raposo A, Santos IHF (2013) Apprc: a framework for integrating mobile communication to virtual reality applications. In: Proceedings of the 12th ACM SIGGRAPH International Conference on VirtualReality Continuum and Its Applications in Industry, VRCAI'13, pp. 305-308. ACM, New York, NY, USA (2013). doi: 10.1145/2534329.2534374.

8. Cruz-Neira C, Sandin DJ, DeFanti TA, Kenyon RV, Hart JC (1992). The cave: audio visual experience automatic virtual environment. Commun ACM 35:64-72. doi:10.1145/129888.129892.

9. P.C. Bell, R.M. O'keefe (1995) An experimental investigation into the efficacy of visual interactive simulation ,Management Science, 41 (6) (1995), pp. 1018-1038

10. R.D. HurrionA sequential method for the development of visual interactive meta-simulation models using neural networks, Journal of the Operational Research Society, 51 (6) (2000), pp. 712-719,CrossRef View Record in Scopus.

11. Herndon, K., van Dam, A., \& Gleicher, M. (1994). The challenges of $3 D$ interaction. SIGCHI Bulletin, 26(4), 36-43.

12. Blender January (1998).Happy Birthday Blender! .Blender Nation. January 2,2014,Retrieve March 29, 2018.

13. Poupyrev, I., Billinghurst, M., Weghorst, S., \& Ichikawa, T. (1996). The Go-Go interaction technique: Non-linear mapping for direct manipulation in VR. Proceedings of the ACM Symposium on User Interface Software and Technology, 79-80.

14. Bowman, D. A., Koller, D., \& Hodges, L. F. (1997). Travel in immersive virtual environments: An evaluation of viewpoint motion control techniques. Proceedings of the Virtual Reality Annual International Symposium, 4552 .

15. Darken, R., \& Cevik, H. (1999). Map usage in virtual environments: Orientation issues. Proceedings of IEEE Virtual Reality, 133-140.

16. Froehlich, B., \& Plate, J. (2000). The Cubic Mouse: A new device for three-dimensional input. Proceedings of ACM CHI.

17. Martin, J. (1998). TYCOON: Theoretical framework and software tools for multimodal interfaces. In John Lee (Ed.), Intelligence and Multimodality in Multimedia Interfaces. AAAI Press.

18. Bowman, D., Wineman, J., Hodges, L., \& Allison, D. (1998). Designing animal habitats within an immersive VE. IEEE Computer Graphics \& Applications, 18(5), 913.

19. $R$. $R$ Development Core Team, R: A Language and Environment for Statistical Computing, 2011.

20. Will Ushert, Pavol Klacanskyt, Frederick Federer, Peer-Timo Bremer, Aaron Knoll, Jeff Yarch, Alessandra Angelucci, and Valerio Pascucci. A Virtual Reality Visualization Tool for Neuron Tracing. IEEE TRANSACTIONS ON VISUALIZATION AND COMPUTER GRAPHICS, VOL. 24, NO. 1, JANUARY 2018.
21. Soffers, Jelly H. M.; Hikspoors, Jill P. J. M.; Mekonen, Hayelom K.; et al. (2015). The growth pattern of the human intestine and its mesenteryBMC DEVELOPMENTAL

BIOLOGY Vol: 15 Article:No. 31 Published: AUG22201 5.

22. Journal of the Korean Society of Design Culture (2017). A Study on $3 D$ Printing Technology Application in Automobile Design -Focused on Scenario Based on 3D Printing Technology Development- Abbr : KSDC 2017, vol.23, no.1, pp. 87-100 (14 pages) UCI : http://uci.or.kr/G704-001533.2017.23.1.054.

23. SchaefferB.GoueseuneC(2003)Syzygy:nativepeclustervr. In:Virtualreality, 2003.Proceedings,IEEE,pp.1522.doilo.1109/VR.2003.1191116.

24. Vrml loader,http//threejs.org/exampleswebgl loader vrml.html.

25. Unity3D:https:unity3d.com/cn/ 